\title{
W. G. Sebald oder der Schwindel der Liebe
}

Lassen Sie mich nach unserer ausführlichen Beschäftigung mit der auf Französisch schreibenden Assia Djebar nun in aller Kürze auf einen deutschsprachigen Text eingehen, auf den 1990 erschienenen Band Schwindel. Gefühle, der von dem 1944 in Wertach im Allgäu geborenen und 2001 bei einem Autounfall tragisch ums Leben gekommenen Schriftsteller W. G. Sebald stammt. Dieser Band enthält sehr unterschiedliche Erzähltexte, die gänzlich verschiedenartigen Themen in variationsreichen narrativen Darstellungen nachgehen, dabei aber immer wieder versuchen, dem Phänomen des Schwindels auf hintergründige, Geschichte und Geschichten respektierende Weise nachzuspüren. Die Thematiken reichen folglich vom Schwindel als Gefühl bis hin zu den Gefühlen als Schwindel, eine fürwahr weite Spanne, die mit hoher literarischer Präzision ausgemessen wird. Dabei geht es selbstverständlich auch um die Liebe als Gefühl und um das Gefühl der Liebe im Schwindel und als Schwindel. Sie merken, wie komplex diese Themenstellung ist, die an dieser Stelle aber leider nicht näher ausgeleuchtet werden kann.

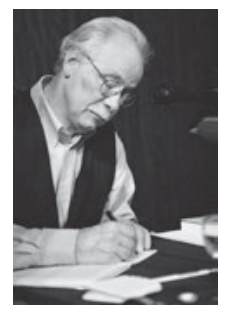

Abb. 56: W. G. Sebald (Wertach im

Allgäu, 1944 - Norfolk, England, 2001).

Der Grund, warum ich mich der Beschäftigung mit W. G. Sebald und diesem Buch zuwende, ist ein vielfacher. Erstens ist es die Thematik des Schwindels in ihrer Beziehung zur Liebe, auf die wir bereits gestoßen sind und auf die wir im weiteren Verlauf unserer Vorlesung erneut treffen werden. Der zweite, spezifisch literaturwissenschaftliche Grund geht darauf zurück, dass sich W. G. Sebald in seinem in der Folge zu analysierenden Text mit fundamentalen Funktionen und Verfahren der Literatur auseinandersetzt und dabei insbesondere die Beziehung zwischen Literatur und Leben in einem engen Verbund sieht, beide Bereiche also miteinander verzahnt. Dabei geht gerade das Nicht-ineinander-Aufgehen mit dem Gefühl des Schwindels einher.

Der dritte Grund für unsere Entscheidung ist die Tatsache, dass die erste Erzählung, die den Band Schwindel. Gefühle eröffnet, jenem Verfasser eines Lehrbuches über die Liebe gilt, der später vor allem als Autor großer Romane wie Le rouge et le noir oder La chartreuse de Parme hervortrat und berühmt wurde. 
Seinen großen Romanen liegt dabei die von ihm entwickelte Liebeskonzeption zu Grunde, mit der wir uns aufgrund ihrer Wirkung unbedingt beschäftigen sollten weit über W. G. Sebald hinaus. Ich spreche von jenem französischen Schriftsteller, der unter dem Namen Henri Beyle geboren und unter seinem Pseudonym Stendhal berühmt geworden ist. Seit Hugo Friedrichs erstmals 1939 erschienener Studie Drei Klassiker des französischen Romans ist dieser Stendhal eingegangen in die heilige Trinität französischer Klassiker, und das Interesse an ihm ist, vergleichbar mit Honoré de Balzac oder Gustave Flaubert, bis heute nicht erlahmt. Das berühmte Buch aber, in welchem Stendhal seine eigene Liebeskonzeption entwickelt und verankert hat, heißt schlicht De l'amour oder Über die Liebe.

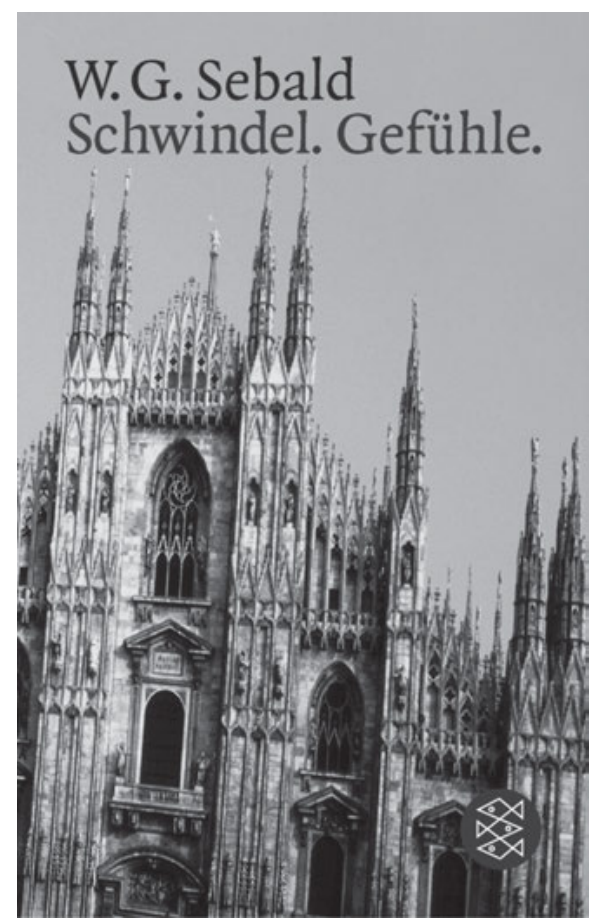

Abb. 57: Cover und Inhaltsverzeichnis von W. G. Sebalds Schwindel. Gefühle.

Diesem Henri Beyle alias Stendhal also ist der erste Erzähltext im Band Schwindel. Gefühle von Sebald gewidmet. Er trägt den Titel „Beyle oder das merckwürdige Faktum der Liebe“. Dies ist zweifellos ein seinerseits höchst merkwürdiger Titel, der schon im Titelbegriff des Faktums auf jene Tatsache hindeutet, die meist nicht ohne einen gewissen Verlust des Bezugs zur Realität und eine überhöhte Beziehung zu der umgebenden Wirklichkeit einhergeht. Kein Wunder also, wenn und dass die Liebe Schwindel erzeugt. Aber ist die Liebe selbst auch ein Schwindel? 


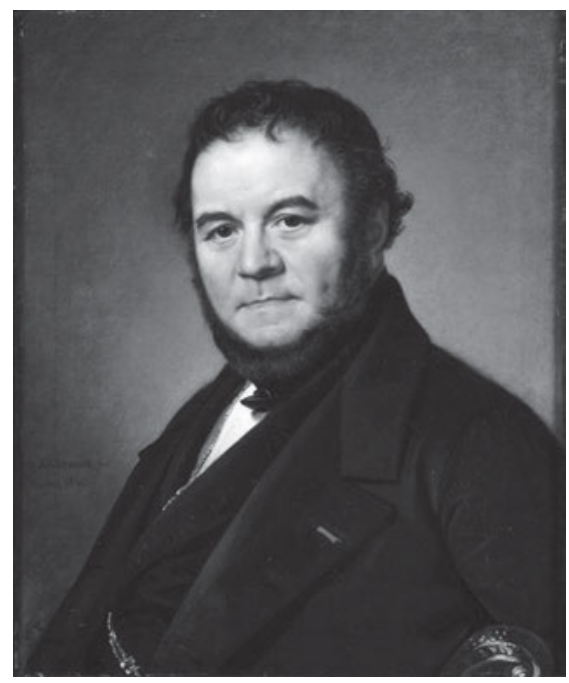

Abb. 58: Stendhal, eigentlich Marie-Henri Beyle (Grenoble, 1783 - Paris, 1842).

Dies darf man sich nach der Lektüre des Textes auch durchaus fragen. Der Erzähltext beginnt mit der Teilnahme des jungen Henri Beyle, der wegen seiner weit auseinanderstehenden Augen auch von seinen Kameraden gerne „le chinois“ genannt wurde, an der berühmt-berüchtigten Alpenüberquerung der napoleonischen Truppen im Jahre 1800. So stand dem Feldherrn Italien offen, ein bis heute in Frankreich liebevoll gepflegter kleiner Mythos.

Damit aber begann zugleich der vielleicht wichtigste Abschnitt im Leben des Henri Beyle: die Liebe zu Italien, die ihn ein Leben lang begleitete und beglückte. Diese Liebe zu Italien rief bei ihm mehr als einmal Schwindel in allen denkbaren Formen hervor. Es war eine Liebe, die auch manche Liebe zu einer hübschen Italienerin miteinschloss.

All diese Erfahrungen machten starken Eindruck auf das sehr intensive, sensible, bisweilen dramatische, ja sogar tragische Liebesempfinden des jungen Franzosen, der unter gelegentlichen Anwandlungen von sexueller Impotenz litt, über die er in stark verhüllter Form auch literarisch schrieb. So kam es bei Beyle zu einer bewegenden Auseinandersetzung mit dem Wesen jenes Gefühls, das wir gewöhnlich „die Liebe“ nennen. Seine Reflexionen, Erlebnisse und Erfahrungen führten schließlich zur Ausprägung jener sogenannten Kristallisationstheorie, die im Zentrum der Stendhal'schen Liebeskonzeptionen steht.

W. G. Sebald betont in seinem zunächst wie ein literaturkritischer Text funktionierenden Erzähltext, dass es sehr wohl möglich sei, dass die schöne junge Frau, die Stendhal auf seiner Reise von Oberitalien über Innsbruck nach Salzburg und in die Salzstollen von Hallein begleitet haben soll, und die zum Entwicklungsobjekt jener besagten Kristallisationstheorie wurde, nur vom französischen 
Schriftsteller imaginiert war und niemals mit dem realen Autor außerhalb des Textes eine Reise in das Salzbergwerk am nördlichen Abhang der Alpen unternommen hat. Aber dies spielt keine wirkliche Rolle; denn Stendhals Theorie entfaltet sich auch am imaginierten weiblichen Objekt - und vielleicht sogar besser als in einer konkreten und nicht vorgestellten Liebesbeziehung. Wir haben es schließlich mit einem hochkarätigen literarischen Autor zu tun, für den die Fiktion eine höhere Form der Wahrheit darstellt.

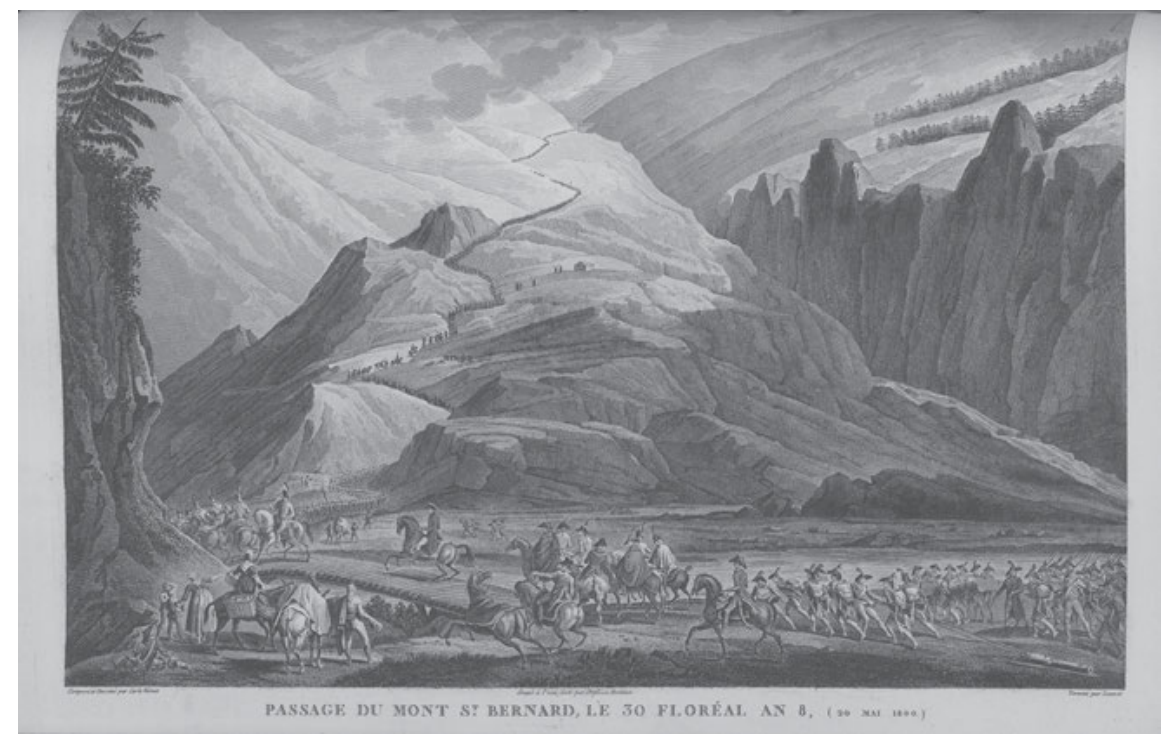

Abb. 59: „Passage du Mont St. Bernard, le 30 floréal an 8“, Radierung von Jean DuplessiBertaux und Louvet nach einer Zeichnung von Carle Vernet.

Der letzte Text von Roland Barthes, den der französische Zeichentheoretiker zu Lebzeiten schrieb und der noch in seiner Schreibmaschine steckte, als man seinen Tod in einem Pariser Krankenhaus feststellte, widmet sich ausgerechnet Stendhal und trägt einen wunderschönen Titel: „On échoue toujours à parler de ce qu'on aime“, „Man scheitert stets, will man von dem sprechen, was man liebt“. In diesem letzten Essay geht es um vieles von dem, was Barthes selbst liebte, aber auch um seine Liebe zu Italien und Stendhal, mit dessen Liebeskonzeption er sich gerade auch in seinen Fragments d'un discours amoureux intensiv auseinandergesetzt hatte. Immer wieder ist der große Romancier des französischen 19. Jahrhunderts zum Gesprächspartner für die verschiedensten Autoren geworden, die sich mit dem Phänomen der Liebe auseinandergesetzt haben: Sebald macht hier also keine Ausnahme. 
Kehren wir daher zu ihm und seiner Beschäftigung mit jener Reise zurück, die den Kristallisationspunkt für die Liebeskonzeption Stendhals bilden sollte. Ausgangspunkt einer Reise, die eigentlich nicht geplant war, sondern relativ spontan erfolgte, ist die Stadt Bologna, die aber unter einer enormen Hitzeglocke lag. So beschlossen der junge Mann und die junge Frau, die freilich kein Liebespaar bildeten, an die oberitalienischen Seen und konkret zum Gardasee auszuweichen - auf der Suche nach etwas Kühlung. Das war damals nicht anders als zu Zeiten des römischen Reichs, als die Seen im italienischen Alpenvorland, eingebettet in malerische Gebirgszüge, ebenfalls gesuchte Erholungsorte waren.

Doch führte diese Reise nicht unbedingt zu einer Kühlung der großen Gefühle. Dafür sorgte schon die junge Frau mit Blick auf den etwas unsicheren Franzosen selbst. Aber immerhin kam es zu einer Kühlung der klimatischen Bedingungen des Sprechens über die Liebe, des Sprechens über das, was man liebt. Aber lesen wir Sebalds Erzähltext, um uns ein genaueres Bild zu verschaffen! Es ist eine gelesene Erzählung, ein gelesenes Erleben, das uns vom Autor als Leser Stendhals gleichsam doppelt gebrochen gespiegelt wird. Erneut stehen also Liebe und Lesen in einer intensiven Wechselbeziehung; und das Bild von Henri Beyle steht für den deutschen Autor im Mittelpunkt einer genauen Lektüre:

Bei Tag rastend, bei Nacht reisend, durchqueren sie das Hügelland der Emilia Romagna und die von schwefligen Schwaden überhangenen Sümpfe von Mantua, um am Morgen des dritten Tages in Desenzano am Gardasee anzulangen. In seinem ganzen Leben nicht, schreibt Beyle, habe er die Schönheit und Einsamkeit dieses Gewässers tiefer empfunden als damals. Der drückenden Hitze wegen hätten er und Mme Gherardi die Abende auf dem See draußen auf einer Barke verbracht und im Einbruch der Dunkelheit die seltensten Abstufungen der Farben gesehen und die unvergeßlichsten Stunden der Stille erlebt. An einem dieser Abende hätten sie sich, so schreibt Beyle, über das Glück unterhalten. Mme Gherardi habe dabei die Behauptung aufgestellt, daß die Liebe, wie die meisten anderen Segnungen der Zivilisation, eine Chimäre sei, nach der es uns umso mehr verlange, je weiter wir uns entfernten von der Natur. In dem Maße, in dem wir die Natur nur in einem anderen Körper noch suchten, kämen wir ab von ihr, denn die Liebe sei eine Leidenschaft, die ihre Schulden in einer von ihr selbst erfundenen Währung begleiche, ein Scheingeschäft also, das man zu seinem Glück ebensowenig brauche wie den Apparat zum Zuschneiden der Federkiele, den er, Beyle, sich in Modena gekauft habe. Oder glauben Sie etwa, so habe sie, schreibt Beyle, noch hinzugefügt, Petrarca sei unglücklich gewesen, nur weil er nie einen Kaffee trinken konnte? ${ }^{1}$

In der romantisch inszenierten Gesprächssituation, in welcher ein freies Sprechen über die intimsten Gefühle möglich wird, erklärt sich die Gesprächspartnerin und gibt ihre Ansicht von der Liebe und damit auch vom Leben kund. In

1 Sebald, W.G.: Schwindel. Gefühle. Frankfurt am Main: Fischer 1990, S. 28 f. 
ihrem berufenen Mund erscheint die Liebe als eine Chimäre - und wir könnten damit schon vermuten, dass sie die Liebe, etwas vulgärer ausgedrückt, für einen einzigen Schwindel hält. Da kommt einem der Refrain eines Hits von Tina Turner in den Sinn: „What's Love but a second hand emotion?“

In dieser spiegelnden Szenerie entwickelt im Erzähltext unter der doppelten Feder von Sebald und Stendhal die junge Frau ihre Ansicht von der Liebe, die diese als ein Produkt der Zivilisation, und damit als ein kulturell erzeugtes Phänomen erscheinen lässt. Liebe ist gerade nicht der menschlichen Natur überantwortet, sondern schillert als ein künstlicher Gegenstand in den Augen seiner Betrachterinnen und Betrachter. Dies gibt zu denken!

Denn diese zivilisatorische Hervorbringung - oder auch Erfindung - der Liebe wird von der Gesprächspartnerin als Chimäre vorgestellt, die eine eigene Ökonomie, einen eigenen Austausch geschaffen habe, welcher zugleich natürlich auch eine eigene Währung erzeugt habe, in der alle Rechnungen in Liebesdingen zu begleichen seien. Die Liebe erscheint hier ganz eindeutig als ein Schwindel in dem Maße, als sie als Zivilisationsprodukt ihre eigene Ökonomie geschaffen habe, die eine Ökonomie des Scheins und gerade nicht eine Ökonomie des Seins sei. Diese Theorie wird in der offenen Szenerie einer romantischen Bergwelt vorgetragen, in einer Szenerie der Zweisamkeit, gleichsam in der Liebessituation überhaupt, in der sich eine heterosexuelle Liebe literarisch auszudrücken vermag. Damit kann natürlich auch jederzeit der Funke der Liebe überspringen und sich entzünden. Anders als Roland Barthes wählt Stendhal nicht die absolute Einsamkeit des liebenden Subjekts, sondern schafft, wohl mit fiktionalen Mitteln, eine heterosexuelle Austauschsituation, von der aus die Thesen und Gegenthesen entwickelt werden können.

Denn dieser Liebestheorie der schönen Madame Gherardi setzt - wie könnte sie auch lange unwidersprochen bleiben? - das männliche Ich schon bald auf der anderen Seite der Alpen seine eigene Gegen-Theorie entgegen. Dafür wählt der französische Schriftsteller ein anderes Setting. Sie wird nicht in der Offenheit der voralpinen Wasserfläche, sondern in der gleichsam fossil überstauten und vor allem abgeschlossenen Höhlendimension des Halleiner Salzbergwerks entwickelt und wenig später dezidiert vor- und ausgetragen. Die jeweiligen Situationen von These und Gegenthese könnten also gegensätzlicher nicht angelegt sein.

Zuvor war die Episode am Gardaseee unschön zu Ende gegangen, da sich die Zeichen des Todes in Riva am oberen Ende des Gardasees gemehrt hatten und sich die junge Dame genötigt sah, fast fluchtartig die Seelandschaft zu verlassen, da diese nun für sie eindeutig im Zeichen des Todes stand. So gelangte das ungleiche Paar also auf die andere Seite der Alpen, aber zugleich auch auf die andere Seite der Geschlechter, auf die andere Seite der offenen oder geschlossenen 
Räumlichkeiten und vor allem auf die andere Seite eines (geteilten) Glücks, das doch in beiden Visionen im Grunde nicht zu haben war. W. G. Sebald arbeitet mit Hilfe seiner Erzählkunst diese Dimension sehr schön heraus und macht zugleich deutlich, dass die Theorie der Liebe von der Praxis der Liebe oft meilenweit entfernt ist und vielfach nichts mit ihr zu tun hat:

Das düstere Ereignis von Riva, das sie in der Erinnerung noch einige Male überlief wie ein Schatten, war bald vergessen und machte einem solchen Übermut Platz, daß sie sich vor lauter Freude in Innsbruck einen breitkrempigen Tirolerhut kaufte, wie wir ihn aus Abbildungen des Hoferschen Aufgebots kennen, und Beyle, der hier eigentlich hatte umkehren wollen, veranlaßte, mit ihr noch weiter das Inntal hinab über Schwaz und Kufstein bis nach Salzburg zu fahren. Dort versäumten sie es während eines mehrtägigen Aufenthalts nicht, die weithin berühmten unterirdischen Galerien des Halleiner Salzbergwerks zu besuchen, wo Mme Gherardi von einem der Mineure ein zwar toter, dafür aber von Tausenden von Kristallen überzogener Zweig zum Geschenk gemacht wurde, an welchem, als sie wieder an den Tag zurückgekehrt waren, die Strahlen der Sonne so vielfach glitzernd sich brachen, wie sonst, so schreibt Beyle, nur das Licht eines hell erleuchteten Ballsaals an den Diamanten der von den Kavalieren im Kreise herumgeführten Damen.

Der langwierige Prozeß der Kristallisation, der den toten Zweig in ein wahres Wunderwerk verwandelt hatte, schien Beyle, wie er eigens ausführt, eine Allegorie für das Wachstum der Liebe in den Salzbergwerken unserer Seelen. Lange redete er, dieses Gleichnis betreffend, auf Mme Gherardi ein. Mme Gherardi war aber nicht bereit, von der kindlichen Seligkeit, die sie an diesem Tag bewegte, abzulassen, um mit Beyle den tieferen Sinn der, wie sie spöttisch anmerkte, zweifellos sehr schönen Allegorie zu erörtern. Beyle empfand dies als eine Demonstration der auf der Suche nach einer seiner Gedankenwelt entsprechenden Frau unvermittelt immer wieder auftauchenden Schwierigkeiten, und er vermerkte, er habe damals eingesehen, daß auch die extravagantesten Veranstaltungen seinerseits diese Schwierigkeiten nicht würden aus dem Weg räumen können. Damit war er bei einem Thema angelangt, das ihn als Schriftsteller auf Jahre hinaus noch beschäftigen sollte. ${ }^{2}$

Henri Beyles Theorie von der langsamen, mühevollen Kristallisation als Prozess der Hervorbringung von Liebe ist berühmt geworden. Ihm gilt unsere volle Aufmerksamkeit. In der Inszenierung des Schriftstellers Stendhal im eigenen Text, und mehr noch in jener Inszenierung Sebalds gelingt diese Allegorie jedoch nicht in ihrer Übersetzung in die Praxis der Liebe. Es ist eine Schwierigkeit, eine Barriere, man könnte auch sagen eine Unfähigkeit oder - um es beim Namen zu nennen - eine Impotenz des Schriftstellers, die hier andererseits zum Ausgangspunkt einer lebenslangen Beschäftigung mit dem Phänomen der Liebe, und damit letztlich mit seinem Werk als Schriftsteller wird. Geradezu obsessiv hat diese Konstellation Stendhal in seinem Schreiben immer wieder begleitet - und

2 Sebald, W.G.: Schwindel. Gefühle, S. 30f. 
eben dadurch ist sie schriftstellerisch so produktiv geworden. Denn Obsessionen sind für Schriftstellerinnen und Schriftsteller oftmals der Ausgangspunkt für eine enorme Kreativität.

Ohne all die Probleme, die wir in unserer kurzen Besprechung lediglich angedeutet haben, hätte es wohl nicht jenen berühmten Schriftsteller gegeben, der sich das Pseudonym Stendhal gab. Die Liebe erweist sich hier auf eine ganz andere Weise als Chimäre: Indem sie nämlich jene Welten erzeugt und bezeugt, die sich in einer eigenen, mit den Mitteln der Fiktion hervorgebrachten Darstellung einer gelebten Realität in der Lektüre entfalten. Sie erreichen ihren Kristallisationspunkt eben in jener Welt, die wir die Welt der Literatur nennen - und sie sind ebenso faszinierend wie jener funkelnde, von Kristallen behaftete Zweig, der seine Banalität auf solch künstlerische Weise transformiert hat und im Licht der Sonne glitzert. In W. G. Sebalds Erzähltext sind Leben, Lieben und Lesen ganz eindeutig enggeführt. Doch machen wir an dieser Stelle zunächst noch einen kleinen Sprung, um weitere Aspekte der abendländischen Liebeskonzeptionen zu erfassen! 